\title{
PHYSICAL AND CHEMICAL PROPERTIES OF POLYCYCLIC AROMATIC HYDROCARBONS
}

\author{
S. LEACH \\ Département Atomes et Molécules en Astrophysique \\ Observatoire de Paris-Meudon \\ 92190 - Meudon, France \\ and \\ Laboratoire de Photophysique Moléculaire du C. N. R.S. \\ Bâtiment 219, Université Paris-Sud \\ 91405 - Orsay, France
}

\begin{abstract}
A review is presented of some physical and chemical properties of polycyclic aromatic hydrocarbons (PAHs) that are relevant for interpreting various aspects of observations and speculations on PAHs in the interstellar medium. The subjects discussed are: the stability and reactivity of neutral and ionic PAHs; the spectroscopy and photophysics of neutral and monocationic PAHs; the photostabilities of PAH monocations and dications and their astrophysical implications.
\end{abstract}

\section{INTRODUCTION}

Polycyclic Aromatic Hydrocarbons (PAHs) in neutral and/or cationic form have been suggested as carriers of a set of interstellar infrared emission bands in the 3-13 $\mu \mathrm{m}$ region (Léger and Puget, 1984; Allamandola, Tielens and Barker, 1985) and also of the Diffuse Interstellar Bands (Van der Zwet and Allamandola, 1985; Léger and d'Hendecourt, 1985). Modelling the formation, excitation and relaxation of the suggested carriers requires information on several aspects of the physics and chemistry of PAHs. The present contribution considers the following:

- the stability and reactivity of neutral and ionic PAHs;

- the spectroscopy and photophysics of neutral and monocationic PAHs;

- the photostability of PAH monocations and dications and astrophysical implications.

\section{STABILITY OF NEUTRAL PAHS}

\subsection{THERMODYNAMIC STABILITY}

Polycyclic aromatic hydrocarbons are conventionally classified as catacondensed or pericondensed. Our discussion will be restricted to the benzenoid varieties of these 
TABLE 1

NUMBER OF BENZENOID PAH ISOMERS

\begin{tabular}{|c|c|c|c|c|c|}
\hline \multirow{2}{*}{$\begin{array}{l}\text { Number } \\
\text { of Rings }\end{array}$} & \multirow{2}{*}{$\begin{array}{c}\text { Formula } \\
\mathrm{C}_{6} \mathrm{H}_{6}\end{array}$} & \multicolumn{2}{|c|}{$\begin{array}{l}\text { Catacondensed (Cata) } \\
\text { or Pericondensed (Peri) }\end{array}$} & \multicolumn{2}{|c|}{$\begin{array}{c}\text { Number of Benzenoid } \\
\text { Isomers }\end{array}$} \\
\hline & & Cata/Peri & $d=2.5 \AA$ & & \\
\hline 2 & $C_{10} H_{10}$ & Cata & & & \\
\hline 3 & $C_{14} H_{10}$ & Cata & & & \\
\hline 4 & $C_{18} H_{12}$ & Cata & & & \\
\hline 5 & $C_{22} H_{14}$ & Cata & & & \\
\hline 6 & $C_{26} H_{16}$ & Cata & & & \\
\hline 7 & $C_{30} H_{18}$ & Cata & & & \\
\hline \multirow[t]{2}{*}{8} & $C_{34} H_{20}$ & Cata & & & \\
\hline & & & & $\begin{array}{l}\text { Non-radical } \\
\text { Isomers }\end{array}$ & $\begin{array}{c}\text { Diradical } \\
\text { Isomers }\end{array}$ \\
\hline 4 & $C_{16} H_{10}$ & Peri & & 1 & 0 \\
\hline 5 & $C_{20} H_{12}$ & Peri & & 3 & 0 \\
\hline 6 & $C_{22} H_{12}$ & Peri & & 2 & 1 \\
\hline $7\left(D_{6 \mathrm{~h}}\right)$ & $C_{24} H_{12}$ & Peri & $d=7.5 \AA$ & 1 & 0 \\
\hline 6 & $C_{24} H_{14}$ & Peri & & 13 & 1 \\
\hline 7 & $C_{26} H_{14}$ & Peri & & 62 & 1 \\
\hline 7 & $C_{28} H_{16}$ & Peri & & 9 & 6 \\
\hline $19\left(D_{6 \mathrm{~h}}\right)$ & $C_{54} H_{18}$ & Peri & $d=12.5 \AA$ & 1 & 0 \\
\hline $37\left(D_{6 \mathrm{~h}}\right)$ & $C_{96} H_{24}$ & Peri & $d=17.5 \AA$ & 1 & 0 \\
\hline $61\left(D_{6 \mathrm{~h}}\right)$ & $C_{150} H_{30}$ & Peri & $d=22.5 \AA$ & 1 & 0 \\
\hline
\end{tabular}

two classes although other types of polyhexes, e. g. coronoids (Balaban et al., 1987; He Wenje et al., 1988) exist. In neutral form the benzenoid PAHs are assumed to be planar but it is very probable that beyond a certain size three dimensional structures are more stable than the planar forms (Haddon, 1988; Frenklach and Ebert, 1988).

A property of some importance, especially in considering PAH formation processes and transformation possibilities, is PAH isomerization. Here one must distinguish between all possible isomers of a particular PAH (for example, benzene has 216 possible isomeric forms of $C_{6} H_{6}$ ) and the possible benzenoid isomers. Table 1 gives the numbers of benzenoid PAH isomers for the first members of a series of catacondensed and pericondensed PAHs. The number of hexagonal rings is indicated, as well as the number of non-radical and diradical pericondensed isomers. Higher spin state PAHs can exist for the larger PAHs (Dias, 1986). In this table are also given properties of some members of the most compact class of pericondensed PAHs, the "circum" PAHs of $D_{6 \mathrm{~h}}$ symmetry built up annularly on benzene. Their diameters $d$ are estimated for planar structures. 
TABLE 2

EQUILIBRIUM MOLE FRACTIONS OF THE BENZENOID PAH $C_{18} H_{12}$

\begin{tabular}{|l|ccccccccc|}
\hline \hline & \multicolumn{10}{|c|}{ Temperature $T / \mathrm{K}$} \\
\cline { 2 - 9 } \multicolumn{1}{|c|}{$C_{18} H_{12}$ Isomer } & 298.15 & 300 & 500 & 700 & 1000 & 1500 & 2000 & 2500 & 3000 \\
\hline Naphthacene & 0.0000 & 0.0000 & 0.0011 & 0.0034 & 0.0064 & 0.0095 & 0.0115 & 0.0130 & 0.0142 \\
Benz(a)anthracene & 0.0033 & 0.0034 & .0387 & 0.0661 & 0.0769 & 0.0794 & 0.0801 & 0.0811 & 0.0824 \\
Benzo(c)phenanthrene & 0.0119 & 0.0125 & 0.2420 & 0.5090 & 0.6829 & 0.7703 & 0.7983 & 0.8099 & 0.8150 \\
Chrysene & 0.0672 & 0.0686 & 0.1773 & 0.1608 & 0.1164 & 0.0830 & 0.0697 & 0.0632 & 0.0596 \\
Triphenylene & 0.9177 & 0.9155 & 0.5409 & 0.2606 & 0.1174 & 0.0579 & 0.0404 & 0.0328 & 0.0287 \\
\hline
\end{tabular}

For PAH formation processes there is, at present, little direct knowledge, some speculation and, more often, benign neglect as to the interstellar context (see however Feigelson and Frenklach, 1989; and Keller, 1987). Two simplistic basic chemical approaches are possible (Omont, 1986): 1) build up of PAHs from smaller hydrocarbon species, and 2) formation of PAHs by hydrogen atom attack and fixation on small graphitic particles which are eventually converted, by physical processes (interstellar shocks, grain-grain collisions, cosmic ray spallation processes, etc.) into PAHs of the required size. A definition of the required size is: not too small so that photodestruction would be faster than infrared emission processes, and not too large so that the infrared emission would be phonon-like, rather than molecular-like, and so occur overwhelmingly in the lowest energy regions of the infrared rather than in the (molecular) 3-13 $\mu \mathrm{m}$ region. Added to these general problems are two requirements that stem from observational constraints and/or interpretations: the PAHs are partially dehydrogenated and are also largely in monocationic form.

One factor which may be of importance in certain PAH formation processes is the isomeric composition of a $\mathrm{PAH}$ mixture if this is formed under conditions of thermodynamic equilibrium. There are expected to be significant modifications in the distribution of benzenoid isomers of a PAH of specific formula $C_{\mathrm{n}} H_{\mathrm{m}}$ over the temperature range $300-3000 \mathrm{~K}$, as can be ascertained from a calculation of the equilibrium mole fractions within isomer groups, based on the Gibbs energy of PAH formation which gives the stability of a particular PAH with respect to graphite and molecular hydrogen (Alberty and Reif, 1988). An illustration of this is given in Table 2 for the group $C_{18} H_{12}$.

\subsection{PHOTOSTABILITY AND REACTIVITY OF NEUTRAL PAHS}

The photostability of gaseous neutral PAHs has been little studied beyond the first member, benzene, which readily isomerizes to a limited number of structures at photon energies around $5 \mathrm{eV}$ and dissociates to various products at higher energies. An important parameter is the limiting size of PAHs beyond which absorption of ultraviolet radiation in $\mathrm{HI}$ regions leads, not to photodissociation, but to the more rapid process of radiative relaxation. The two models employed to describe the competing processes are discussed elsewhere in this volume. Although the solid state inspired "classical model" (Léger and Puget, 1984; Puget, Léger and Boulanger, 1985) of internal energy redistribution is physically less appealing than the quantum mechanical-based RRKM/Quasiequilibrium Theory (QET) approach 
(Allamandola, Tielens and Barker, 1985), recent work shows that they give essentially the same rate predictions for large PAHs at high levels of excitation (Barker and Cherchneff, 1989; d'Hendecourt et al., 1989). This is not surprising in view of the statistical mechanics parameters involved in both models. Thus it is predicted that vibrational radiative relaxation to attain energy levels below the lowest dissociation limit, following absorption of a $10 \mathrm{eV}$ photon, will be substantially faster than the rate of atomic evaporation (solid state model) or of attaining a dissociative nuclear configuration (RRKM/QET model), for PAHs containing a minimum of number of carbon atoms, variously 20-30 (Allamandola, Tielens and Barker, 1985), 20-40 (Ryter, Puget and Pérault, 1987), 40 (Désert et al., 1987), 50 (Léger and Puget, 1984), or 100 (Puget, Léger and Boulanger, 1985). However it must be stressed that the photostability of PAHs in these size ranges has never been tested experimentally. This is one of several urgent tasks in this field.

Aihara (1987), working within the context of the graph theory of aromaticity (Trinajstic, 1983), has discussed the photochemical stability of PAHs. He uses resonance energy as an index of thermodynamic stability, and then makes the very questionable assumption that the photochemical stability of the first excited state is related to thermodynamic stability to argue that large compact PAHs, containing 14 aromatic rings or more, are the most photostable. It seems to me more useful to limit this approach to the ground electronic state of PAHs, in particular with respect to resistance to chemical attack.

The reactivity of PAHs probably involves edges, defects and other active sites. Stein and Brown (1987) have examined the correlation between edge structures and quantum chemical and thermodynamic parameters related to reactivity, for hexagonally symmetric PAHs containing up to 2300 carbon atoms. Hückel molecular orbital theory was used as the basis for calculations. The main parameters studied as a function of PAH size and edge structure were resonance energy per electron, maximum free valence and heat of formation. The results showed that edge structure and overall size of PAHs should be critically important factors in determining $\mathrm{PAH}$ reactivity. Fully benzenoid molecules are expected to be generally not very reactive, consistent with known experimental reactivities of smaller PAHs (Clar, 1972). The chemical attack of PAHs by atoms $(H, O, N, \ldots)$ could occur in the interstellar medium. The rates are generally unknown (nevertheless, see Duley and Williams (1986)) but should be sensitive to the physical and chemical nature of the site attacked.

\section{SPECTROSCOPY AND PHOTOPHYSICS OF NEUTRAL AND MONOCA- TION PAHS}

\subsection{NEUTRAL PAHS}

Hydrocarbons are conveniently divided into two types, alternant and non-alternant hydrocarbons. In an alternant hydrocarbon alternate carbon atoms can be starred, with no starred atoms adjacent to each other, example: naphthalene, Fig. 1. If adjacent starred atoms ensue, then the system is non-alternant, example: azulene, Fig. 1. Coulson and Rushbrooke (1940) showed that alternant $\pi$-bonded hydrocarbons will always be molecules with a self-consistent field since the same average number of $\pi$-electrons exists at each carbon atom, i.e., the total $\pi$-electron charge density is spread uniformly over the carbon atoms. The alternant hydrocarbons 
NAPHTHALENE

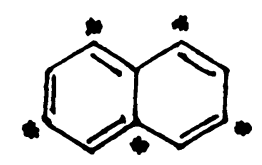

(EVEN) ALTERNANT HYDROCARBON

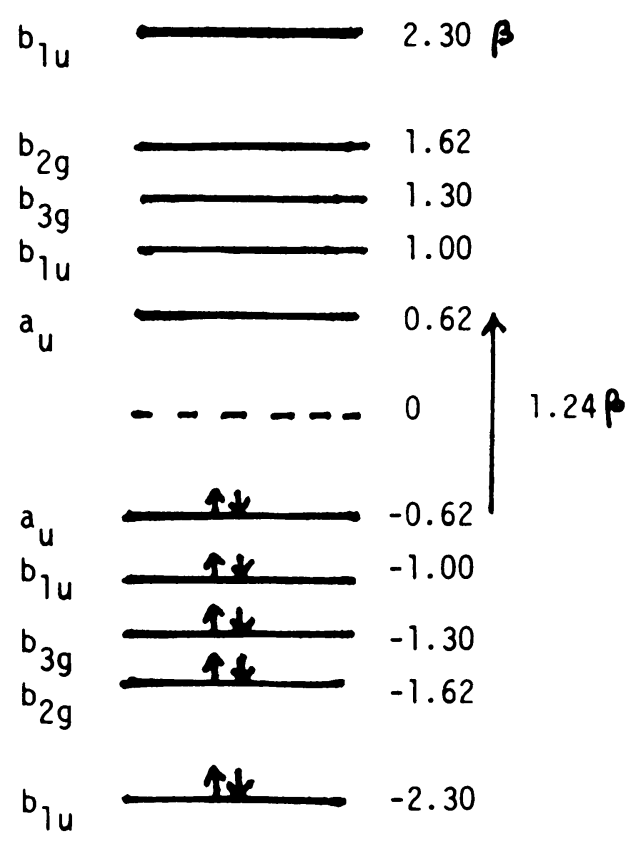

\section{AZULENE}

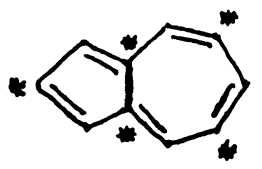

NON-ALTERNANT HYDROCARBON

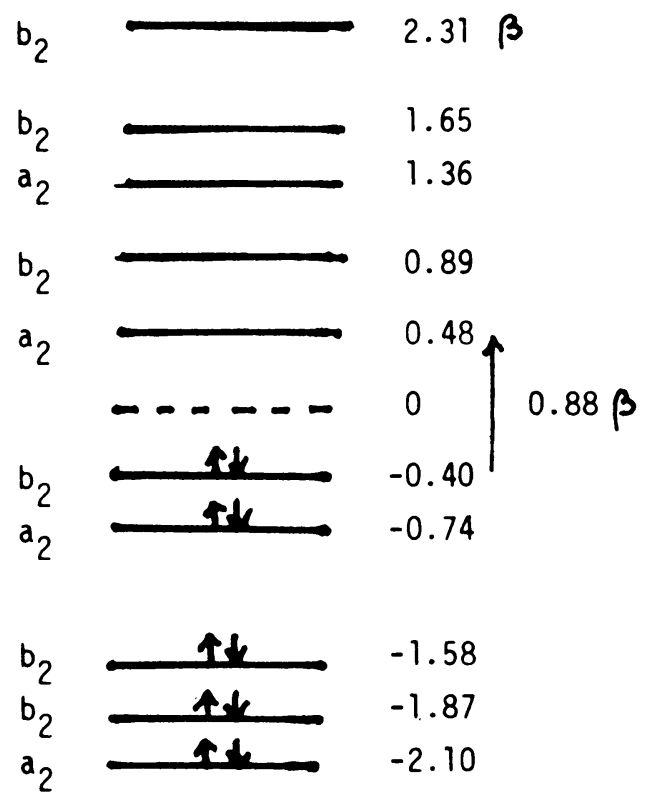

Fig. 1. One-electron $\pi$ molecular orbitals for naphthalene and azulene, illustrating characteristics of alternant and non-alternant hydrocarbons. 
are characterized by the property that for every bonding molecular orbital (M.O.) of energy: $-E$, there exists a complementary antibonding orbital of energy: $+E$; the paired (conjugate) bonding and antibonding M.O.'s are arranged symmetrically about a zero energy reference.

Alternant hydrocarbons are either even or odd. In an even-alternant, the number of carbon atoms, $N_{c}$, is even and the numbers of starred and unstarred atoms are generally equal, so that there are $N_{c} / 2$ bonding and $N_{c} / 2$ antibonding orbitals. If an odd-alternant, $N_{c}$, is odd, and there is usually one more starred carbon atom than unstarred, one nonbonding orbital is found between the pairs of conjugate orbitals. The even-alternant species are particularly stable since each of the $N_{c} / 2$ bonding orbitals are occupied by two electrons (small arrows in Fig. 1) and none of the nonbonding orbitals. The uniform charge distribution makes the hydrocarbon relatively resistant to chemical attack since no centres of attraction exist for potential reagents to attach themselves.

The molecular orbital energies and symmetries can be calculated by various methods. A Hückel method calculation provides information on one-electron energy levels whose energies are expressed in terms of the resonance integral $\beta$ which is essentially the energy of an electron in the fields of two neighbouring carbon atoms which are bonded together. The value of $\beta$ can be determined by various methods; for spectral transition calculations $\beta \approx-2.7 \mathrm{eV}$; a semi-empirical value of $\beta$ can be obtained from its dependence on carbon-carbon bond distance (Mulliken, Rieke and Brown, 1941; Salem, 1966).

The one-electron energy levels are given in Fig. 1 for the even-alternant hydrocarbon naphthalene and its non-alternant isomer azulene. The results predict that ratio of the lowest optical transition energy in azulene to that of naphthalene should be about 0.71 . This is true for the second singlet-singlet transition of both species. This, and further detailed comparison with experimental transition energies reveals the effects of configuration interaction, going beyond the simple Hückel molecular orbital one-electron picture. species.

The situation for naphthalene is illustrated in Fig. 2 which presents one-electron energy levels, electron occupations, and optical transitions for alternant hydrocarbons. This simple model leads to a more correct description of the four lowest energy singlet-singlet transitions. The two highest occupied ground state molecular orbitals (M. O.) are labelled $i, j$; the two lowest unoccupied M. O.'s are $k, l$. The labels $\chi_{0}, \chi_{0}^{k}$, etc., represent the various electron configurations corresponding to the M.O. electron occupancies in Figure 2. Four optical transitions can occur as illustrated in the Figure. Two common notations used for the resulting bands, transitions and excited states, are those of Clar (1941) and Platt (1949). The most correct notation would be that of the symmetry of the electronic wavefunction of a particular state in a species belonging to a given point-group. However, the Clar and Platt notations are very useful for rapidly recognizing spectral resemblances among hydrocarbon

Figure 2 shows that the $p$ and $\beta^{\prime}$ bands are normally expected to have respectively the lowest and highest energies of the four transitions. Since $\chi_{j}^{k}$ and $\chi_{i}^{l}$ differ in energy, any configuration interaction involving them would be of minor consequence for the $p$ and $\beta^{\prime}$ transitions. The other two transitions, corresponding to the $\alpha$ and $\beta$ bands, are degenerate in energy at the one-electron level; for example, 


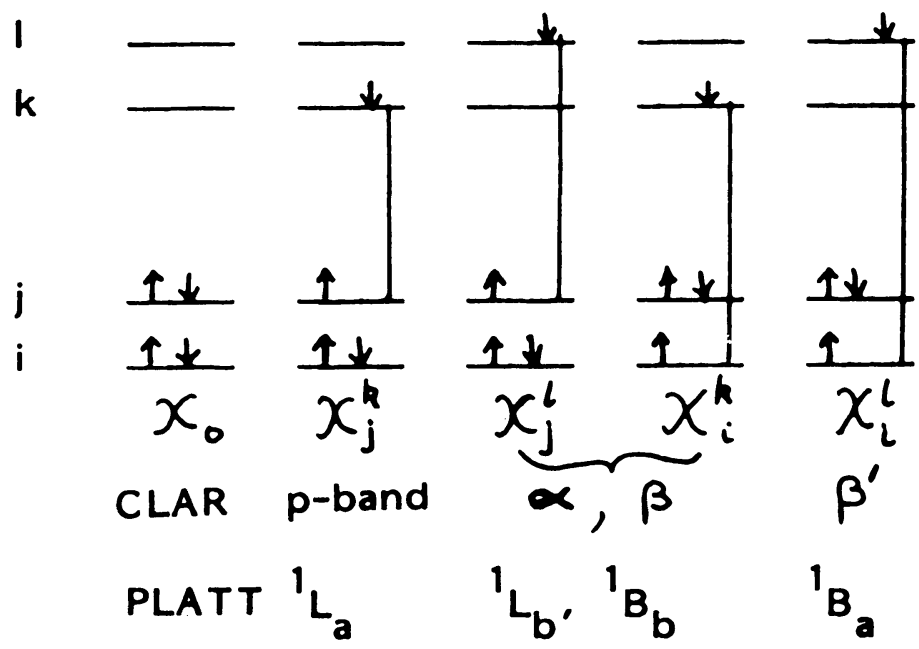

Fig. 2. Lowest energy transitions and band notations for alternant hydrocarbons.

for naphthalene, the $\alpha$ and $\beta$ transitions would each have an energy of 1.62 $\beta$ (Fig. 1). The $\chi_{j}^{l}$ and $\chi_{i}^{k}$ configurations form two linear combinations, one plus, the other minus, which then correlate with the $\alpha$ and $\beta$ states. This configuration interaction can be sufficiently strong, in certain PAH cases, for the $\alpha$ band to lie at lower energies than the $p$ band.

Absorption transitions to the $p$ state are expected to be weak, those to the $\boldsymbol{\beta}^{\prime}$ state are usually relatively strong. Configuration interaction causes the transition moments to cancel in the minus linear combination of $\chi_{j}^{l}$ and $\chi_{i}^{k}$, so that the $\alpha$ band is usually very weak and often has, as its major component, terms involving Herzberg-Teller type vibronic coupling. In contrast, the $\beta$ transition moments add in the positive linear combination of $\chi_{j}^{l}$ and $\chi_{i}^{k}$. For PAHs the intensities and oscillator strengths of the four transitions can be characterized as follows: $\alpha$ : very weak, $f \approx 0.001 ; p$ : weak, $f \approx 0.01-0.1 ; \beta$ : very strong, $f \approx 1 ; \beta^{\prime}$ : medium strong, $f \approx 0.01-1$. These oscillator strengths are valid for catacondensed PAHs and suffer some exceptions for pericondensed PAHs, as gleaned from the data published by Clar (1964). Data for some catacondensed and some pericondensed PAHs are given in Tables 3 and 4 respectively. The $\beta^{\prime}$ bands are not assigned for the pericondensed species; transitions (bands) above $\beta$ are designed $S_{4}, S_{6}, S_{6}$. 
TABLE 3

WAVElengths AND Oscillator StREngths, $f$, For some CATACONDENSED PAHS

\begin{tabular}{|l|c|c|c|c|c|c|c|c|}
\hline \hline \multirow{3}{*}{ PAH } & \multicolumn{9}{c|}{ Band/Transition } \\
\cline { 2 - 9 } & \multicolumn{2}{|c|}{$\alpha$} & \multicolumn{2}{c|}{$p$} & \multicolumn{2}{c|}{$\beta$} & \multicolumn{2}{c|}{$\beta^{\prime}$} \\
\cline { 2 - 9 } & $\lambda /(\mathrm{nm})$ & $f$ & $\lambda /(\mathrm{nm})$ & $f$ & $\lambda /(\mathrm{nm})$ & $f$ & $\lambda /(\mathrm{nm})$ & $f$ \\
\hline Benzene $C_{6} H_{6}$ & 263 & 0.002 & 210 & 0.10 & 185 & 0.69 & see $\beta$ & see $\beta$ \\
Naphthalene $C_{10} H_{8}$ & 313 & 0.003 & 289 & 0.18 & 220 & 1.70 & 170 & 0.80 \\
Azulene $C_{10} H_{8}$ & 695 & 0.009 & 364 & 0.08 & 296 & 1.10 & 193 & 0.65 \\
Pentacene $C_{22} H_{14}$ & 417 & 0.005 & 585 & 0.10 & 313 & 2.20 & - & - \\
\hline
\end{tabular}

TABLE 4

WAVelengths AND EXtinction COefficients $\log \epsilon$ FOR SOME PERICONDENSED PAHS

\begin{tabular}{|c|c|c|c|c|c|c|c|c|c|c|c|c|}
\hline \multirow{3}{*}{ PAH } & \multicolumn{12}{|c|}{ Band/Transition } \\
\hline & \multicolumn{2}{|c|}{$\alpha$} & \multicolumn{2}{|c|}{$p$} & \multicolumn{2}{|c|}{$\beta$} & \multicolumn{2}{|c|}{$S_{4}$} & \multicolumn{2}{|c|}{$S_{5}$} & \multicolumn{2}{|c|}{$S_{6}$} \\
\hline & $\lambda / \mathbf{n m}$ & $\log \epsilon$ & $\lambda / \mathbf{n m}$ & $\log \epsilon$ & $\lambda / \mathrm{nm}$ & $\log \epsilon$ & $\lambda / \mathrm{nm}$ & $\log \epsilon$ & $\lambda / \mathrm{nm}$ & $\log \epsilon$ & $\lambda / \mathbf{n m}$ & $\log \epsilon$ \\
\hline Pyrene $C_{16} H_{10}$ & 370 & 2.71 & 335 & 4.74 & 272 & 4.73 & 241 & 4.94 & 207 & 4.59 & - & - \\
\hline Perylene $C_{20} H_{12}$ & 292 & 3.15 & 435 & 4.60 & 252 & 4.72 & 263 & 4.04 & 227 & 4.34 & 206 & 4.93 \\
\hline Coronene $\mathrm{C}_{24} \mathrm{H}_{12}$ & 420 & 2.70 & 353 & 4.23 & 302 & 5.45 & 228 & 3.90 & 215 & 4.74 & 200 & 5.15 \\
\hline Ovalene $C_{32} H_{14}$ & 465 & 4.15 & 457 & 4.68 & 348 & 5.26 & - & - & - & - & - & - \\
\hline
\end{tabular}

units for $\epsilon$ are $l / m o l e / c m$

Good correlations between the energies of the $p, \alpha, \beta$ and $\beta^{\prime}$ transitions and properties related to the first two ionization potentials have been found by Schmidt (1977) for a series of 61 catacondensed aromatic hydrocarbons. These relations can be rationalized in terms of the general self-consistent field properties of alternant hydrocarbons, with corrections for electron repulsion and configuration interaction. It was also shown that reactivity (e.g. ease of photo-oxidation, maleic anhydride addition) in the acene series parallels ionization properties which are related to the amount of configuration interaction between $\chi_{i}^{l}$ and $\chi_{i}^{k}$.

The spectral information on PAH transition energies and intensities has mostly been obtained from solution spectra; it is thus subject to condensed phase interactions and inhomogeneous broadening phenomena. Laser induced fluorescence excitation and dispersed fluorescence studies in the gas phase have been carried out on a number of PAHs of interstellar interest using supersonic jet cooled techniques. Coronene (Bermudez and Chan, 1986, 1987; Babbitt, Ho, and Topp, 1988) and ovalene (Amirav, Even, and Jortner, 1981) are among the most interesting cases investigated. Lifetimes and line shape features reflecting vibrational and vibronic coupling effects have been studied. These high resolution studies are restricted to the first and second excited electronic states of the species studied. A quantumchemical analysis of the absorption and emission spectra of coronene has recently been carried out by Orlandi and Zerbetto (1988).

The absorption spectra of benzene and naphthalene have been studied in the gas phase over the range 5 to $30 \mathrm{eV}$ (Koch and Otto, 1972; Koch, Otto and Radler, 
1972). Useful information can also be obtained from electron energy loss spectroscopy. Much of the work on the gas phase species pertains to Rydberg states observed at relatively low resolution. Much higher resolution studies could undoubtedly be carried out in absorption on PAHs using the new spectrometers designed for synchrotron radiation work in a number of facilities.

Increasing energy of photon excitation beyond the absorption threshold leads to the formation of a variety of stable and dissociative valence and Rydberg states. Coupling can exist between these various states, giving rise to peculiarities in the intramolecular dynamics. The Rydberg states above the first ionization potential (I. P.) can have an additional relaxation, autoionization, which provides ways of reaching the ionization continuum in addition to that of direct ionization. A measure of the importance of nonionic relaxation channels of superexcited states is given by the photoionization efficiency $\gamma(I)$, defined as the number of ions produced per photon absorbed. For molecules, $\gamma(I)$ does not reach unity until several volts above the ionization threshold e.g. for benzene, whose I. P: ${ }^{\prime} 9.24 \mathrm{eV}, \gamma(I)=1$ at 16.53 $\mathrm{eV}$.

\subsection{IONIC PAHS}

In this section will be presented a discussion of data on electronic states of PAH cations and on their intramolecular relaxation channels, contrasted and compared with those for neutral PAHs. However, it is convenient first to give a brief discussion of the ion chemistry of PAHs in interstellar clouds.

\subsubsection{Ion Chemistry of PAHs in Interstellar Clouds}

The build-up, and to a greater extent, the state of existence and the destruction of PAHs in interstellar clouds, have been discussed in the literature, with particularly important contributions from Duley and Williams (1981), Duley (1986), Omont (1986), Lepp and Dalgarno (1988), and Lepp, Dalgarno, Van Dishoeck and Black (1988). PAHs are estimated to be mainly in cationic or anionic form in various parts of the interstellar medium (ISM). In what follows the reaction rates related to ions and ionization processes have mainly been taken from these authors.

In dense clouds ionization will mainly be by charge transfer from existing atomic and molecular ions formed via cosmic ray ionization. Charge transfer should be a high yield process in view of the low ionization potential $(\approx 6.5 \mathrm{eV})$ of PAHs. High rates (e. g. Langevin rates $\approx 10^{-9} \mathrm{~cm}^{3} \mathrm{~s}^{-1}$ can be expected since the multitude of conjugated $\pi$-electrons should make the PAHs very polarizable. PAH monocations can disappear by dissociative recombination with electrons $\left(k \approx 6 \times 10^{-6}(100 / T)^{-1 / 2}\right.$ $\mathrm{cm}^{3} \mathrm{~s}^{-1}$ ), by recombination on grain surfaces, or by reaction (mutual neutralization) with PAH or other anions $\left(k \approx 10^{-6} T^{-1 / 2} \mathrm{~cm}^{3} \mathrm{~s}^{-1}\right)$. PAH anions can be formed by electron attachment. This reaction $\left(k \approx 8 \times 10^{-7} \mathrm{~cm}^{3} \mathrm{~s}^{-1}\right)$ can be dissociative or lead to a stable anion; the electron affinity of PAHs is considered to be of the order of $2 \mathrm{eV}$. The destruction of PAH anions can occur by mutual neutralization $(k \approx$ $\left.{ }^{10^{-6}} T^{-1 / 2} \mathrm{~cm}^{3} \mathrm{~s}^{-1}\right)$. Collisions involving neutral and ionic PAHs could lead to the building up of larger molecular systems.

In diffuse clouds, ionization will mainly be by photoionization $(\sigma \approx 100 \mathrm{Mb})$. 
KOOPMANS STATES

I

k
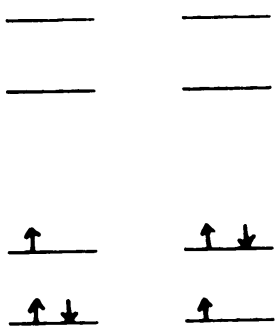

j

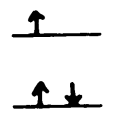

$D_{0}$
1

$D_{1}$
NON-KOOPMANS STATES
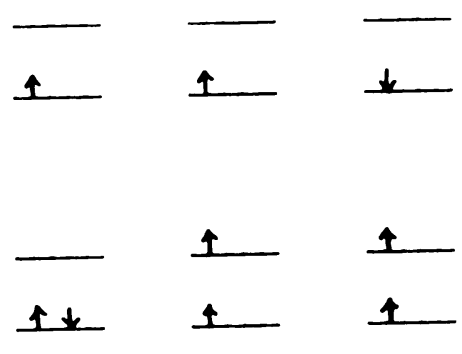

$\mathrm{D}_{2}$ $\perp$

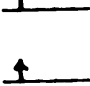

1

$D_{3}$

Fig. 3. Koopmans and non-Koopmans states and configurations for molecular ions.

The incident photons could rapidly lead to electron loss of PAH anions by photodetachment which should also have a relatively high cross-section.

\subsubsection{Spectroscopy and Photophysics of PAH Monocations}

Most of the information on the spectroscopy of PAH monocations comes from absorption spectra of PAH ions formed by photolysis or radiolysis in solid matrices and also from photoelectron spectroscopy of gas phase PAHs. There are very few calculations of PAH cation energy levels carried out on the ions themselves. Most relevant calculations are on the neutral PAH M.O. energies which also provide information on ionic states if Koopmans theorem (Koopmans, 1934) is assumed to be valid.

The cation states observed by photoelectron spectroscopy can be described as Koopmans states since they can be reached by one electron transitions from the fully occupied orbitals of the neutral PAH (Fig. 3). Non-Koopmans states would require two-electron transitions; these states can be reached by optical transitions from Koopmans states in the cation (Fig. 3). Extensive data on the photoelectron spectra of PAHs has been obtained by Schmidt and his colleagues (Clar, Robertson, Schlögl and Schmidt, 1981 and earlier references cited). The lowest lying cation states are $\pi$ states; the first $\sigma$ state occurs at an energy of about $3-4 \mathrm{eV}$ in the ion (Boschi, Clar, and Schmidt, 1974).

The absorption spectra of both anions and cations of a series of PAHs has been observed in a 2-methyl-tetrahydrofuran and other glassy matrices at $77 \mathrm{~K}$ after $\gamma$-ray irradiation of the PAH solutes (Shida and Iwata, 1973). Similar cation spectra have been obtained by Kahn $(1984,1987,1988)$ in a boric acid matrix at room temperature; the cations were formed by photoionization of the PAH solutes. Comparison with calculated band energies and intensities were made in both sets of studies, and correlations made between the spectra of the various ions. The cation and anion spectra of a given benzenoid PAH are quite similar, especially 
in the visible region, as expected from the conjugate nature of the M.O.'s. The cation absorptions consist of a series of bands in the visible and ultraviolet. For the typical case of the coronene cation, for example, there are several bands in the visible, followed by very strong absorption bands $(f \approx 1)$ in the 360 and $290 \mathrm{~nm}$ regions, and a yet stronger broad peak at $215 \mathrm{~nm}$ (Khan, 1988).

All of the PAH monocations under discussion in the present work are openshell species. The energy separation $\Delta E\left(D_{1}-D_{0}\right)$ between the ground electronic state (doublet state $D_{0}$ ) and the first excited state of the same multiplicity (double state $\left.D_{1}\right)$ is less than in the nearest related closed-shell species. This is illustrated in a tabulation given elsewhere (Leach, 1987) which lists the energy of the first excited state relative to the ground state of same multiplicity for some open-shell cationic hydrocarbons and their parent neutral closed-shell species. For example, for coronene, the $S_{1}-S_{0}$ transition is at $2.90 \mathrm{eV}$; for its monocation, the $D_{1}-D_{0}$ transition is at $1.29 \mathrm{eV}$. Ions of structure having symmetry axes of order greater or equal to 3 (e.g. benzene cation and coronene cation) can have orbitally degenerate electronic states and so exhibit spontaneous Jahn-Teller distortion from the most symmetrical configuration. This must be taken into account in evaluating properties of PAH ions of interest to the ISM.

The $D_{1}$ state in open-shell ions will tend to lie below the first dissociation limit, whereas in the corresponding closed-shell parent, the first excited singlet state often has an energy close to, or greater than, that of the lowest dissociation threshold, and therefore could be more subject to predissociation. Furthermore, the lower excitation energy of the open-shell species brings the first electronic transition to a more easily accessible spectral region from the viewpoint of excitation (at least under laboratory circumstances) and detection of photon emission.

The polarizability of PAHs, in neutral or ionic form, should play a role in the possibility of them forming complexes and as an important factor in their chemical reactivity. The polarizability $\alpha$ of a PAH ground state can be expressed as a weighted sum of electronic transition moments:

$$
\alpha=2 \sum_{m \neq 0} \frac{\left|R_{m o}\right|^{2}}{E_{m 0}}
$$

where $R_{m o}$ is the transition dipole moment for the transition to excited state $m$. As has been discussed above, the lowest excited electronic states of PAH cations and anions are at smaller energies than for neutral PAHs. This implies that $\alpha$ can be larger for PAH ions than for neutral PAHs. The contribution of the manifold of states above the ionization limit is expected to increase with decreasing ionization potential, so this part of $\alpha$ will be expected to be greatest for the anion, less for the molecule, and least for the cation. When all factors are taken into account, both simple M.O. theory and $a b$ initio calculation (Hinchcliffe, Munn, and Siebrand, 1983) predict that the polarizability of an alternant hydrocarbon cation will often be in the order anion $>$ cation $>$ neutral molecule.

Another property of open shell species is particularly relevant to the applicability of radiationless transition theories, and provides a simplification with respect to closed-shell systems. The excited $S_{1}$ state can be coupled to vibronic levels of at least two lower-lying electronic states, namely, the first excited triplet state $T_{1}$ and the electronic ground state $S_{0}$. However, in the open-shell species of concern to us, 
the vertical excitation energy with respect to the $D_{0}$ state of the lowest-lying quartet state $Q_{1}$ will be greater than that of $D_{1}$, so that coupling leading to radiationless transitions will occur between $D_{1}$ and vibronic levels of only one lower-lying state, i.e. the electronic ground state $D_{0}$, as discussed elsewhere (Leach, 1987).

Intramolecular electronic nonradiative transitions fall into a number of cases which reflect increasing density of interacting levels. A brief presentation will be given here only for the statistical limit case, which would apply to large species such as aromatic hydrocarbons.

The optically excited zero-order state $\mid s>$ (e.g. $D_{1}$ for open shell and $S_{1}$ for closed shell species) is coupled nonradiatively to vibrational states $\{\mid l>\}$ of a lower electronic level. For ions, the matrix element $v_{s l}$ involved in electronic nonradiative transitions will generally involve only the nuclear kinetic energy operator, (for closed shell species, and in general where the transition involves spin multiplicity changes, there will be contributions from spin-orbit operators). In the statistical limit, the density of $\{\mid l>\}, \rho_{1} \gg 10^{5}$ states $/ \mathrm{cm}^{-1}$, so that the $\{\mid l>\}$ states form a quasicontinuum. Electronic nonradiative transitions are then virtually irreversible so that the Fermi-Wentzel Golden Rule radiationless rate is given by the expression $k_{n r}=(2 \pi / \hbar) \rho_{l} v_{s l}^{2}$ when $v_{s l}$ varies slowly enough to be represented by its average value. The decay of the $|s\rangle$ state is then mono-exponential.

The relaxation of several molecular ions has been studied within the statistical limit context. For the benzene ion $k_{n r}$ was found to be greater than $8 \times 10^{10} \mathrm{~s}^{-1}$ for the $\tilde{B}^{2} E_{2 g}$ state and greater than $5 \times 10^{12} \mathrm{~s}^{-1}$ for $\tilde{C}^{2} A_{2 u}$ (Braitbart, Castellucci, Dujardin and Leach, 1984). These excited states decay by $\tilde{C}, \tilde{B} \rightarrow \tilde{X}$ interelectronic state coupling and by isomerisation to Dewar benzene and/or benzvalene cations. Recent vibronic coupling calculations have confirmed the proposed $\tilde{C}, \tilde{B} \rightarrow \tilde{X}$ coupling mechanism (Köppel, Cederbaum and Domcke, 1988). The high vibrational levels in the ground state of benzenoid ions, which are the final states of interelectronic state coupling in these species, might be involved in isomerization processes in some cases. Otherwise the other most probable collisionless relaxation process would be infrared radiative emission. This appears to have a rate of the order of $10 \mathrm{~s}^{-1}$ for the benzene ion ground state containing 2.4-2.7 eV vibrational energy (Dunbar, 1986). Indeed a study of several polyatomic ions by different techniques (Van Velzen and Van der Hart, 1981; Dunbar, 1983) indicates that the collisionless relaxation of $1-2 \mathrm{eV}$ of internal energy occurs at rates of the order of $1-100 \mathrm{~s}^{-1}$. Still higher rates have been suggested for infrared radiative relaxation of vibrational levels at $3.5 \mathrm{eV}$ above the zero-vibration level of the ground state of other cations (Leach, Dujardin and Taieb, 1980).

\subsubsection{Thermodynamic Stability and Photostability of PAH Cations}

Experimental data on the heats of formation of PAH monocations is very sparse (Rosenstock, Drexl, Steiner, and Herron, 1977), but methods of estimation exist. Using these methods, Stein (1983) has shown that protonated and ionized benzenoid PAHs are more stable than any of their nonbenzenoid structural isomers.

As stated earlier, the most consistent data on the ionization potentials of PAHs come from photoelectron spectroscopy, especially from the work of Schmidt (Clar, Robertson, Schlögl and Schmidt, 1981, and earlier references cited in this article). 
Representative first ionization potentials up to hexabenzocoronene $\mathrm{C}_{42} \mathrm{H}_{18}$ have been tabulated (Leach, 1986). The values for species larger than naphthalene are all less than $8 \mathrm{eV}$ and rapidly approach about $6.5 \mathrm{eV}$ for higher PAHs. Indeed, large polycyclic aromatic hydrocarbons have been shown to ionize either like twodimensional sheets, or like linear fragments of graphite. Semi-empirical calculations of ionization energies (Smith, 1961; Wacks, 1964) show that the first ionization potentials of PAHs tend to approach the sum of the graphite work function and the energy necessary to charge a single-plate capacitor whose size and shape is that of the molecule. The appropriate value of the work function to be used in such calculations is structure dependent (Becker and Wentworth, 1963; Wacks, 1964). Examination of the first ionization potential of PAHs as a function of the number of $\pi$ electrons, and organized into different point-group symmetries, makes more clear the trends towards limiting values (Gallegos, 1968). The most recent published data on PAH ionization potentials confirm that of all the PAH molecules, the slowest approach to the solid graphite work function limit of $4.39 \mathrm{eV}$ is for the $D_{3 \mathrm{~h}}$ and $D_{6 \mathrm{~h}}$ species, i.e., for the most symmetrical molecules. An increased rate of approach to limiting value occurs for more linear species. The limiting values for linear PAHs appears to correspond to the work function of a graphite wire (Wacks, 1964), whereas a higher limit value is appropriate to species that are more like twodimensional graphite sheets, such as coronene, ovalene, hexabenzocoronene, etc ... Deviations from the symmetry classification trends occur for the $D_{2 \mathrm{~h}}$ species perylene, pyrene and ovalene, whose ionization energies are higher than expected; this has been rationalized in terms of a tendency to radial or linear structure (Gallegos, 1968).

The photostability of gaseous PAH monocations has been little discussed and has been even less a matter of experimental investigation apart from the benzene cation (Baer, 1986; Kühlewind, Kiermeier, Neusser and Schlag, 1987; Kiermeier, Kühlewind, Neusser, and Schlag, 1988) and the naphthalene monocation (Ruhl, Price, and Leach, 1988). Ion photodissociation has been used on a few small PAHs as a probe for spectroscopic studies (Kim and Dunbar, 1980; Syage and Wessel, 1987).

The study of the photostability of the naphthalene monocation was carried out over the photon energy range 8-35 eV using the threshold photoelectron-photoion coincidence (T-PEPICO) technique (Ruhl, Price and Leach, 1988). Some of the results may be of general interest for the higher PAH cations most likely to be found in the interstellar medium. Analysis of the threshold photoelectron spectrum indicated considerable autoionization to high vibrational levels of the ground state of the monocation. This could be a factor in creating the physical conditions required for application of quasi-equilibrium statistical theory in the fragmentation of naphthalene ion and possibly as a general process in aromatic and other ions. Fragment thresholds and branching ratios observed in the dissociative photoionization of naphthalene were interpreted in terms of thermochemical data, energy barriers and possible sequential reactions. Evidence was found for triple-product dissociation in the higher range of photon energies. Another result of interest is that infrared radiative relaxation is most probably the dominant relaxation process for internal energies of the monocation less than 3-5 eV. For larger PAHs expected to be present in the interstellar medium, the fragmentation thresholds would be expected to occur at rather higher internal energies relative to naphthalene because of the bigger densities of states in the larger molecular ions. Future work in this area will seek to determine the fragmentation thresholds for larger PAH monoca- 
tions and to estimate whether there could be significant photofragmentation of these monocations in $\mathrm{HI}$ regions under interstellar medium conditions.

\subsubsection{PAH Dications}

The lowest dissociation limit of a molecular dication is generally below the double ionization threshold; the lowest dissociation products are two singly-charged ions (Coulomb dissociation). Covalent dissociation to a doubly charged fragment and neutral fragment(s) can also occur and can also have a relatively low dissociation limit with respect to the parent dication threshold (Leach, Eland, and Price, 1989a). Thus molecular dications will tend to be fragile species so that direct dissociation and dissociation via tunneling should be common processes for them.

The possible formation of PAH dications in the interstellar medium has been proposed and discussed by Leach $(1986,1987)$ in connection with the interpretation of the infrared emission bands between 3 and $13 \mu \mathrm{m}$ observed in interstellar HI regions (strongest near the ionization front) and quasi-absent within HII regions. The observations indicate that PAHs are partially dehydrogenated; the ambient interstellar conditions are such that the PAHs could be in cationic form up to $100 \%$ in extent (Omont, 1986). However, fragmentation of PAHs has been considered to be an exceedingly rare process in HI regions, the average lifetime of a PAH species being estimated to be comparable to that of most molecular clouds ( $>10^{6}$ years) (Omont, 1986).

A scheme involving the formation of PAH dications in the interstellar medium and leading to the partial dehydrogenation of PAHs in the HI region, and to their complete destruction in HII regions, has been proposed by Leach $(1986,1987)$. HI and HII circumstellar regions are differentiated by the $\mathrm{H}^{+}-e^{-}$recombination rate being respectively greater and less than that of $H$ atom ionization; the effective upper limit of available photon excitation energy is thus $13.6 \mathrm{eV}$ within the HI region.

An efficient way of forming $\mathrm{PAH}^{2+}$ in the ISM is a sequential two-stage excitation process $\mathrm{PAH}+h \nu_{1} \rightarrow \mathrm{PAH}^{+}+e^{-} ; \mathrm{PAH}^{+}+h \nu_{2} \rightarrow \mathrm{PAH}^{2+}+e^{-}$. The efficiency of the sequential processes results from the expectation that PAHs are readily singlyionized with radiation of less than $13.6 \mathrm{eV}$ energy and that for larger members of these species the ionization energy to form $\mathrm{PAH}^{2+}$ from $\mathrm{PAH}^{+}$could also be less than $13.6 \mathrm{eV}$.

These expectations have been examined in detail (Leach, 1986) for a series of PAHs. The data show that the first ionization potentials $I^{+}$are all well below $13.6 \mathrm{eV}$ and that for the larger PAHs the difference $I^{2+}-I^{+}$is indeed also less than $13.6 \mathrm{eV}$. It was concluded that in the interstellar medium, sequential two photon excitation involving photons of energy less than $13.6 \mathrm{eV}$ would lead to the formation of PAH dications with only a few eV $(\leq 3 \mathrm{eV})$ of internal energy. These are then expected to undergo hydrogen loss dissociation processes but not complete destruction. The physical conditions in the relevant parts of the interstellar medium (Omont, 1986) are compatible with the excitation processes proposed. The more energetic photons in the HII region are expected to lead to their photodestruction. These conjectures have been tested by experiments on a small PAHs, naphthalene, azulene, etc. The results and their astrophysical implications are reported elsewhere (Ruhl, Price, and Leach, 1988; Leach, Eland, and Price, 1989a, 1989b). 
The experiments were carried out using threshold photoelectron-photoion coincidence (T-PEPICO) (Ruhl, Price and Leach, 1988) and photoelectron-photoionphotoion triple coincidence (PEPIPICO) (Leach, Eland and Prince, 1989a, 1989b) techniques using either synchrotron radiation or rare-gas lamp high energy photon sources. Some of the astrophysical implications of the results are summarized below, using the experimental data on naphthalene as an example.

HII Regions: It was shown (Leach, Eland, and Price, 1989a) that the relative yield of doubly-charged to singly-charged naphthalene cations is about $25 \%$ at $\lambda 304 \AA$. The rapid rise in this relative yield with photon energy and, as observed in electron impact studies (Gallegos, 1968), the greater relative yields expected for larger PAHs, indicate that in the high photon energy HII regions there will be extensive formation of doubly-charged PAH cations by direct double photoionization. The PEPIPICO result on naphthalene show that dications of this PAH are extensively destroyed at photon energies of $34.8 \mathrm{eV}(356 \AA)$ and $40.8 \mathrm{eV}(304 \AA)$ by charge separation reactions. The results also indicated a small percentage of stable dications formed at these energies, with lifetimes greater than $\approx 10^{-1} \mathrm{~s}$, under the experimental conditions. Under interstellar conditions even in HII regions, collisions are virtually absent, but stable dications could still relax dissociatively by tunneling through to Coulomb repulsion (charge separation) potential surfaces. However, tunneling would be in competition with infrared emission, whose characteristic lifetimes are $10^{-2}-1 \mathrm{~s}$. These values are several orders of magnitude greater than the lower limit of the lifetime of stable dications formed in the PEPIPICO experiments, so tunneling should dominate under HII cosmic conditions. PAHs are therefore expected to be destroyed in HII regions, via charge separation reactions following double ionization, thus explaining the absence in these regions of infrared emission bands assigned to PAHs.

HI Regions: Partial dehydrogenation of $\mathrm{PAH}$ and $\mathrm{PAH}^{+}$is postulated in $\mathrm{HI}$ regions from interpretation of the infrared emission spectra. As stated above, the astrophysical model involving sequential double ionization of PAHs in HI regions (Leach, 1986) indicates that the PAH dications so formed would have only up to 3 $\mathrm{eV}$ of internal energy $E_{\text {int }}^{2+}$. From the PEPIPICO result on naphthalene, substantial charge separation is not expected in $\mathrm{PAH}^{2+}$ with $E_{\text {int }}^{2+} \leq 3 \mathrm{eV}$. Covalent dissociation of $\mathrm{PAH}^{2+}$ will occur in this internal energy interval, as predicted from the naphthalene results and as has been observed for other PAHs. For example, in anthracene, $\mathrm{C}_{14} \mathrm{H}_{10}$, electron impact studies (Mathur, Burgess, Bostwick, and Moran, 1981) have shown that the appearance potential of the dication fragment $C_{14} H_{8}^{2+}$ is only $0.1 \mathrm{eV}$ above that of the parent dication $C_{14} H_{10}^{2+}$. There are similar observations for higher PAHs, thus providing mechanisms for hydrogen loss in accordance with the astrophysical model.

$\mathrm{C}_{2} \mathrm{H}_{2}^{+}$and $\mathrm{C}_{3} \mathrm{H}_{3}^{+}$Ionic Products: It is striking that $\mathrm{C}_{2} \mathrm{H}_{2}^{+}$and $\mathrm{C}_{3} \mathrm{H}_{3}^{+}$are the major ionic products of naphthalene dication charge separation reactions. These are also the major products for higher PAH dications (Klingston, Gilhaus, Brenton, and Beynon, 1985). This may have astrophysical implications for the formation of certain interstellar molecules, in particular cyclopropenylidene $\mathrm{C}_{3} \mathrm{H}_{2}$ and carbon chain molecules (Leach, Eland, and Price, 1989b).

Neutral Products: The small neutral products of charge separation reactions, $H$, $\mathrm{H}_{2}, \mathrm{C}_{2} \mathrm{H}_{2}, \mathrm{C}_{2} \mathrm{H}_{3}, \mathrm{C}_{4} \mathrm{H}_{2}$, and of covalent dissociations of naphthalene dications are 
also important molecules or intermediates in interstellar chemistry (Winnewisser and Herbst, 1987). Higher PAH dications would be expected to form the same major neutral product species. The possible role of PAH dications as sources of these species merits further consideration.

One further aspect of possible interest is that in charge separation processes considerable kinetic energy is often released which, if statistically distributed, would be taken up mainly by the smaller ionic and neutral products if these are formed in simultaneous three body dissociations. Such kinetic energy could help in overcoming endothermic barriers in some cases of ion-molecule reactions. However, the PEPIPICO results on naphthalene indicate that most three-product reactions occur by sequential deferred charge separation mechanisms rather than by simultaneous fragmentation. This is probably also true for larger PAHs but remains to be tested.

Whether the various astrophysical implications of T-PEPICO and PEPIPICO results on naphthalene discussed in this section are applicable to larger PAHs, and in an interstellar context, requires not only more laboratory work on larger PAHs, but also extensive spectroscopic and cartographic observations of the infrared emission bands and of species that can aid in defining the physico-chemical conditions in appropriate regions of the interstellar medium. Fine grained spatial resolution observations would be of great assistance in this respect.

\section{REFERENCES}

Aihara, J. 1987, Bull. Chem. Soc. Japan, 60, 3143.

Alberty, R. A. and Reif, A. K. 1988, J. Phys. Chem. Ref. Data, 17, 241.

Allamandola, L. J., Tielens, A. G. G. M., and Barker, J. R. 1985, Ap. J., 290, L25.

Amirav, A., Even, U., and Jortner, J. 1981, J. Chem. Phys., 74, 3745.

Babbitt, R. J., Ho, C. J., and Topp, M. R. 1988, J. Phys. Chem., 92, 2422.

Baer, T. 1986, Adv. Chem. Phys., 64, 111.

Balaban, A. T., et al. 1987, Z. Naturfor8ch, 42a, 863.

Barker, J. R. and Cherchneff, I. 1989, in IAU Symposium 195, Interstellar Dust, eds. L. J. Allamandola and A. G. G. M. Tielens, (Dordrecht: Kluwer), p. 197.

Becker, R. S., and Wentworth, W. E. 1963, J. Am. Chem. Soc., 85, 2210.

Bermudes, G., and Chan, I. Y. 1986, J. Phys. Chem., 80, 5029.

1987, J. Phys., Chem., 91, 4710.

Boschi, R., Clar, E., and Schmidt, W. 1974, J. Chem. Phys., 60, 4406.

Braitbart, O., Castellucci, E., Dujardin, G., and Leach, S. 1983, J. Phys. Chem., 87, 4799.

Clar, E. 1941, Aromatische Kohlenwasserstoffe, (Berlin: Springer).

. 1964, Polycyclic Hydrocarbons, (New York: Academic). 1972, The Aromatic Sextet, (London: Wiley), Chap. 6.

Clar, E., Robertson, J. M., Schlögl, R., and Schmidt, W. 1981, J. Am. Chem. Soc., $103,1320$.

Coulson, C. A., and Rushbrooke, G. S. 1940, Proc. Camb. Phil. Soc., 86, 193.

Désert, F. X., Léger, A., d'Hendecourt, L., and Boissel, P. 1987, unpublished, cited by Ryter, Puget and Pérault 1987.

d'Hendecourt, L. B., Léger, A., Boissel, P., Désert, F. X. 1989, in IAU Symposium 195, Interstellar Dust, eds. L. J. Allamandola and A. G. G. M. Tielens, (Dordrecht: Kluwer), p. 207.

Dias, J. R. 1986, J. Molec. Structure (Theochem), 187, 9.

Duley, W. W. 1986, in Polycyclic Aromatic Hydrocarbons and Astrophysics, ed. A. Léger, L. d'Hendecourt and N. Boccara, (Dordrecht: Reidel), p. 373.

Duley, W. W. and Williams, D. A. 1981, M. N. R. A. S., 196, 269. 1986, M. N. R. A. S., $219,859$.

Dunbar, R. C. 1983, J. Phys. Chem., 87, 3105.

1986, Chem. Phys. Lett., 125, 543. 
Feigelson, E. D., and Frenklach, M. 1989, in Interstellar Dust Contributed Papers, eds. A. G. G. M. Tielens and L. J. Allamandola, NASA CP-3036.

Frenklach, M., and Ebert, L. B. 1988, J. Phys. Chem., 92, 561.

Gallegos, E. J. 1968, J. Phys. Chem., T2, 3452.

Haddon, R. C. 1988, Acc. Chem. Res., 21, 243.

He, W. J., et al. 1988, Z. Naturforsch., 43a, 693.

Hinchcliffe, A., Munn, R. W., and Siebrand, W. 1983, J. Phys. Chem., 87, 3837.

Keller, R. 1987, in Polycyclic Aromatic Hydrocarbons and Astrophysics, eds. A. Léger, L. d'Hendecourt and N. Boccara, (Dordrecht: Reidel), p. 387.

Khan, Z. H. 1984, Z. Naturforsch, 39a, 668 .

- 1987, Z. Naturforsch, 42a, 91. 1988, Spectrochim. Acta, 44A, 313.

Kiermeier, A., Kühlewind, H., Neusser, H. J., and Schlag, E. W. 1988, J. Chem. Phys., 88, 6182.

Kim, M. S. and Dunbar, R. C. 1980, J. Chem. Phys., 72, 4405.

Kingston, R. G., Guilhaus, M., Brenton, A. G., and Beynon, J. H. 1985, Org. Mass Spectrom., 20, 406.

Koch, E. E., and Otto, A. 1972, Chem. Phys. Lett., $12,476$.

Koch, E. E., Otto, A. and Radler, K. 1972, Chem. Phys. Lett., 16, 131.

Koopmans, T. 1934, Physica, 1, 104.

Köppel, H., Cederbaum, L. S., and Domcke, W. 1988, J. Chem. Phys., 89, 2023.

Kühlewind, H., Kiermeier, A., Neusser, H. J. and Schlag, E. W. 1987, J. Chem. Phys., 87, 6488.

Leach, S. 1986, J. Electron Spectr. Rel. Phen., 41, 427.

Leach, S. 1987, in Polycyclic Aromatic Hydrocarbons and Astrophysics, eds. A. Léger, L. d'Hendecourt and N. Boccara, (Dordrecht: Reidel), p. 99.

Leach, S., Eland, J. H. D. and Price, S. D. 1989a J. Phys. Chem., submitted.

. 19896 J. Phys. Chem., submitted.

Léger, A., and Puget, J. L. 1984, Astr. Ap., 187, L5.

Léger, A., and d'Hendecourt, L. 1985, Astr. Ap., 146, 81.

Lepp, S., and Dalgarno, A. 1988, Ap. J., 324, 553.

Lepp, S., Dalgarno, A., Van Dishoeck, E. F. and Black, J. H. 1988, Ap. J., 329, 418.

Mathur, B. P., Burgess, E. M., Bostwick, D. E. and Moran, T. F. 1981, Org. Mass Spectrom., 16, 92.

Mulliken, R. S., Rieke, C. A. and Brown, W. G. 1941, J. Am. Chem. Soc., 68, 41.

Omont, A. 1986, Astr. Ap., 164, 159.

Orlandi, G., and Zerbetto, F. 1988, Chem. Phys., 123, 175.

Puget, J. L., Léger, A. and Boulanger, F. 1985, Astr. Ap., 142, L19.

Rosenstock, H. M., Draxl, K., Steiner, B. W., and Herron, J. T. 1977, J. Phys. Chem. Ref. Data, 6, 1.

Rühl, E., Price, S. D. and Leach, S. 1988, J. Phys. Chem., submitted.

Ryter, C., Puget, J. L. and Pérault, M. 1987, Astr. Ap., 186, 312.

Salem, L. 1966, The Molecular Orbital Theory of Conjugated Systems, (New York: Benjamin), chap. 3.

Schmidt, W. 1977, J. Chem. Phys., 66, 828.

Shida, T., and Iwata, S. 1973, J. Am. Chem. Soc., 95, 3473.

Smith, F. T. 1961, J. Chem. Phys., 84, 793.

Stein, S. E. 1983, Combustion and Flame, 51, 357.

Stein, S. E. and Brown, R. L. 1987, J. Am. Chem. Soc., 109, 3721.

Syage, J. A., and Wessel, J. E. 1987, J. Chem. Phys., 87, 3313.

Trinajstic, N. 1983, Chemical Graph Theory, (Boca Raton: CRC Press) Vol. II.

Van der Zwet, G. P. and Allamandola, L. J. 1985, Astr. Ap., 146, 76.

Van Velzen, P. N. T., and Van der Hart, W. J. 1981, Chem. Phys., 61, 325.

Wacks, M. E. 1964, J. Chem. Phys., 41, 1661.

Winnewisser, G. and Herbst, E. 1987, Topics Curr. Chem., 139, 119. 